\title{
Patterns of episodic content and specificity predicting subjective memory vividness
}

\author{
Rose A. Cooper ${ }^{1 *} \&$ Maureen Ritchey ${ }^{2}$ \\ ${ }^{1}$ Department of Psychology, Northeastern University \\ ${ }^{2}$ Department of Psychology and Neuroscience, Boston College \\ * Correspondence concerning this article should be addressed to Dr. Rose Cooper, email: \\ r.cooper@northeastern.edu
}

\section{Abstract}

The ability to remember and internally represent events is often accompanied by a subjective sense of "vividness". Vividness measures are frequently used to evaluate the experience of remembering and imagining events, yet little research has considered the objective attributes of event memories that underlie this subjective judgment, and individual differences in this mapping. Here, we tested how the content and specificity of event memories support subjectively vivid recollection. Over three experiments, participants encoded events containing a theme word and three distinct elements - a person, a place, and an object. In a memory test, memory for event elements was assessed at two levels of specificity - semantic gist (names) and perceptual details (lure discrimination). We found a strong correspondence between memory vividness and memory for gist information that did not vary by which elements were contained in memory. There was a smaller, additive benefit of remembering specific perceptual details on vividness, which, in one study, was driven by memory for place details. Moreover, we found individual differences in the relationship between memory vividness and objective memory attributes primarily along the specificity dimension, such that one cluster of participants used perceptual detail to inform memory vividness whereas another cluster were more driven by gist information. Therefore, while gist memory appears to drive vividness on average, there were idiosyncrasies in this pattern across participants. When assessing subjective ratings of memory and imagination, research should consider how these ratings map onto objective memory attributes in the context of their study design and population. 


\section{Introduction}

The subjective experience of recalling a past event, defined by autonoetic consciousness (Tulving, 2002), is often accompanied by a sense of "vividness". In research on episodic memory and imagination, this subjective quality is used frequently as an indicator of memory ability or imagery strength, where participants are typically asked to rate the vividness with which they can bring an event to mind along a scale from low to high. Vividness judgments have been employed to characterize trial-specific variability in recalling episodic memories (e.g., Cooper et al., 2019; Folville, D’Argembeau, et al., 2020; Robin et al., 2016; Thakral et al., 2019; Xie \& Zhang, 2017), and are a succinct way to understand the neural correlates of recollection (e.g., Bone et al., 2020; Bonnici et al., 2016; Ford \& Kensinger, 2016; Geib et al., 2017; Kuhl \& Chun, 2014; Lee et al., 2018; Richter et al., 2016; Sheldon \& Levine, 2013; Sreekumar et al., 2018; St-Laurent et al., 2015; Tibon et al., 2019). As illustrated by this broad collection of research, "vividness" as a composite judgment of memory quality, richness, or detail can be useful as a catch-all measure in an experimental context. However, as an inherently subjective experience, such judgments may be driven by different memory attributes depending on the individual and context. Considering the frequency with which vividness is used as a memory and imagery measure, surprisingly little research has considered the objective attributes of internal event memory representations that underpin this subjective judgment (for a discussion, see Folville, Simons, et al., 2020).

Episodic memories are highly variable in terms of their content and specificity, as well as in their subjective experience. Imagine meeting a friend for lunch and trying to remember that event the following week - although this could be a simple event to bring to mind, and a memory that feels vivid, the event contains a number of distinct elements (e.g., the specific friend involved, the time and place, as well as associated objects, emotions, conversations, thoughts, etc.), each of which can be represented in memory with varying levels of specificity (e.g., remembering specific visual details about your friend's outfit or the restaurant). While it might be assumed that subjective ratings of memory correspond uniformly to the amount of event information recalled, the relationship between objective and subjective attributes of memory is often weak (Clark \& Maguire, 2020) and can vary substantially between individuals and populations, for example, showing a reduced correlation in healthy aging (Folville, D'Argembeau, et al., 2020). Above and beyond variation in the overall correspondence between objective and subjective memory, the 
subjective vividness of memory might be differentially associated with distinct attributes of event memories, such as the types of elements contained in memory and the specificity with which they are remembered. Here we ask, what are the dominant attributes of episodic memories that we subjectively reexperience as vivid?

We are able to remember an incredible amount of detail about scenes and objects (Bainbridge et al., 2019; Brady et al., 2008), but different kinds of content may be prioritized when remembering a complex event and thus differentially influence subjective vividness. Theoretical accounts of episodic memory have tended to place spatial context as the center of memory retrieval, providing a key scaffold upon which other episodic information can be built (Herweg et al., 2020; Horner et al., 2016; Robin, 2018). For example, Robin and colleagues have found that spatial context serves as a stronger memory cue than a person when remembering event scenarios, and that participants are more likely to spontaneously add a spatial context than a person to a memory if the event did not originally contain that element (Robin et al., 2016). Neurologically, this research is centered on the hippocampus playing an important role in the construction of space and scenes (Hassabis \& Maguire, 2007; Maguire et al., 2016), interacting with neocortical sensory regions to reactivate perceptual details associated with a spatial context (Sekeres et al., 2018; Staresina et al., 2013). Spatial context is also the dominant feature in the neural representation of events (Robin et al., 2018). It stands to reason that judgments of memory vividness may be grounded in memory for place information more so than memory for other elements of the event, but what level of specificity supports a vivid memory? A recent study of individual differences found that self-reported detailed autobiographical memory was more closely tied to object imagery ability (imagining specific colors and shapes of components within scenes) than to spatial imagery ability (imagining spatial relations between objects) (Fan et al., 2020). Therefore, vividness may be more strongly tied to the recollection of specific, perceptual details within a scene rather than a schematic spatial framework.

Less is known about how vividness corresponds to the specificity of individual details within complex event memories. Most studies of multi-element events test memory for gist-level information only, for example, by presenting words of trial-unique person-object-place combinations and asking participants to imagine or remember events containing the triplet (Addis et al., 2009; Horner \& Burgess, 2013; Robin et al., 2018; Thakral et al., 2019). While binding 
gist-level information together is an important foundation and dominant characteristic of episodic memory (Horner \& Burgess, 2013), recalling low-level, perceptual detail appears to be an independent process (Cooper \& Ritchey, 2019; Ngo et al., 2020) that may facilitate a feeling of reliving. Two recent studies have directly investigated the trial-level contributions of memory for low-level perceptual information of scenes to judgments of vividness. In one experiment, Bone and colleagues used layers of a convolutional neural network to study neural reactivation of scene features, finding that both low-level and high-level image features predicted trial-specific judgments of memory vividness (Bone et al., 2020). In another experiment, participants were presented with scenes in which the low-level features of brightness and color saturation were manipulated along a continuous scale (Cooper et al., 2019), and memory vividness could be predicted by how precisely those low-level scene features were recalled. Moreover, overlap in neural activity between perception and imagery tracks subjective vividness primarily in early visual brain regions (Dijkstra et al., 2017). Therefore, while binding and constructing information at a gist level, particularly within a spatial context, is likely to be crucial to remembering an episodic memory vividly, there may be an additive value of recalling perceptual details tied to the individual event elements.

Over three experiments, we examined the relative contribution of different kinds of memory content (people, objects, and places) and their remembered specificity (gist or detailed) to subjective ratings of memory vividness. In Experiments 1 and 2, we used trial-level correlations as well as predictive linear regression models to reveal the memory attributes (content and specificity) that explain the most variance in vividness ratings. In Experiment 3, we additionally manipulated attention to different kinds of event elements during encoding. Overall, we predicted that gist and detail memory would additively contribute to memory vividness, and that this relationship would be particularly strong for place information. Finally, across the experiments, we conducted a clustering analysis to test for individual differences in the pattern of relationships between memory attributes and subjective vividness. 


\section{Experiment 1}

\section{Methods}

The data and analysis code for all experiments are available through our github repository: https://github.com/memobc/paper-vividness-features.

\section{Participants}

A total of 48 participants were included in Experiment 1 analyses. We initially recruited 51 participants from Amazon Mechanical Turk (MTurk) and 27 participants from the Boston College Sona community. After thorough data quality checks (detailed in the Statistical Analyses section), 30 participants were excluded (3 Sona and 27 MTurk), which was 38\% of the total sample, leaving a final sample size of 48 (24 male, 24 female, mean age $=26.7$ years, $\mathrm{SD}=8.7, \min =18, \max =$ 47). Sona participants made up $50 \%$ of the final sample. All participants reported learning English by the age of 6 . Informed consent was obtained from all participants, who were reimbursed for their time. Procedures were approved by the Boston College Institutional Review Board.

\section{Task and stimuli}

The experimental tasks were programmed using PsychoPy (Peirce et al., 2019); https://www.psychopy.org/) and deployed online using Pavlovia (https://pavlovia.org/). After recruitment through either Sona or MTurk, participants were directed to complete the full experiment online. First, they were asked to complete the consent form as well as a basic demographics questionnaire. Participants were then provided with detailed visual and auditory instructions for the main task (see Figure 1). Participants completed one study phase and one test phase, each with 24 trials. Each study trial ("event”) contained a theme word (e.g., 'weekend', 'celebration', 'relaxing', 'volunteer') that could be used to guide the context of an event, and would be subsequently used as the retrieval cue. Around this theme word, participants saw 3 images each paired with a word label: a famous person, an object, and a place. Participants had up to 15 seconds to study the three elements (image + label) and create a meaningful story linking them together around the theme word, and to press the spacebar when they had come up with a story.

The images were collated from various sources, including pairs of similar famous faces from google searches, similar object pairs from the Mnemonic Similarity Task (Kirwan et al., 2007), and 
similar place pairs from (Cooper et al., 2017). All pairs of objects and places depicted perceptually similar versions of, conceptually, the same object/place. Each famous face pair depicted the same person but at a different time, with a different appearance. The background of objects and faces was removed, and all images were cropped and resized to $300 \times 300$ pixels. Twenty-four pairs of people, objects, and places were used such that each was uniquely associated with a single event. The elements were randomly assigned to one of the 24 events, but the combination of elements into events was fixed across participants.

Participants were presented with only the study phase instructions before completing all study phase trials. Immediately after completion of the study phase, participants were presented with the instructions for the test phase. On each test trial, participants were presented with a theme word as a cue for the event to remember. They were first asked to rate the subjective vividness with which they could remember the entire event (all of the elements associated with the theme word and the story they created) on a scale of 1 (not at all vividly) to 6 (extremely vividly). Participants were encouraged to think carefully about the event and take as long as they needed to respond. Next, they were tested on their "gist memory" for the 3 elements. Each gist memory question presented the participant with 4 word labels (either 4 people, objects, or places they had studied) and participants were asked to select which word was associated with the theme. Finally, participants were tested on their "detail memory" for the 3 elements. Each detail memory question presented the participant with two perceptually similar exemplars of the person, object, or place that was associated with the theme (note that images were always based on the correct gist-level association even if the participant answered the corresponding gist memory question incorrectly). Participants were asked to choose which image had been presented in the event. The order of questions for person/object/place was counterbalanced across trials but was fixed for gist and detail questions within the same trial. All test question responses were self-paced. Study and test phase trial orders were randomly generated for each participant. After completing the test phase, participants were redirected to complete two final questionnaires about their memory and mental imagery - the Survey of Autobiographical Memory (Palombo et al., 2013) and the Vividness of Visual Imagery Questionnaire (Marks, 1973). 


\section{ENCODING}
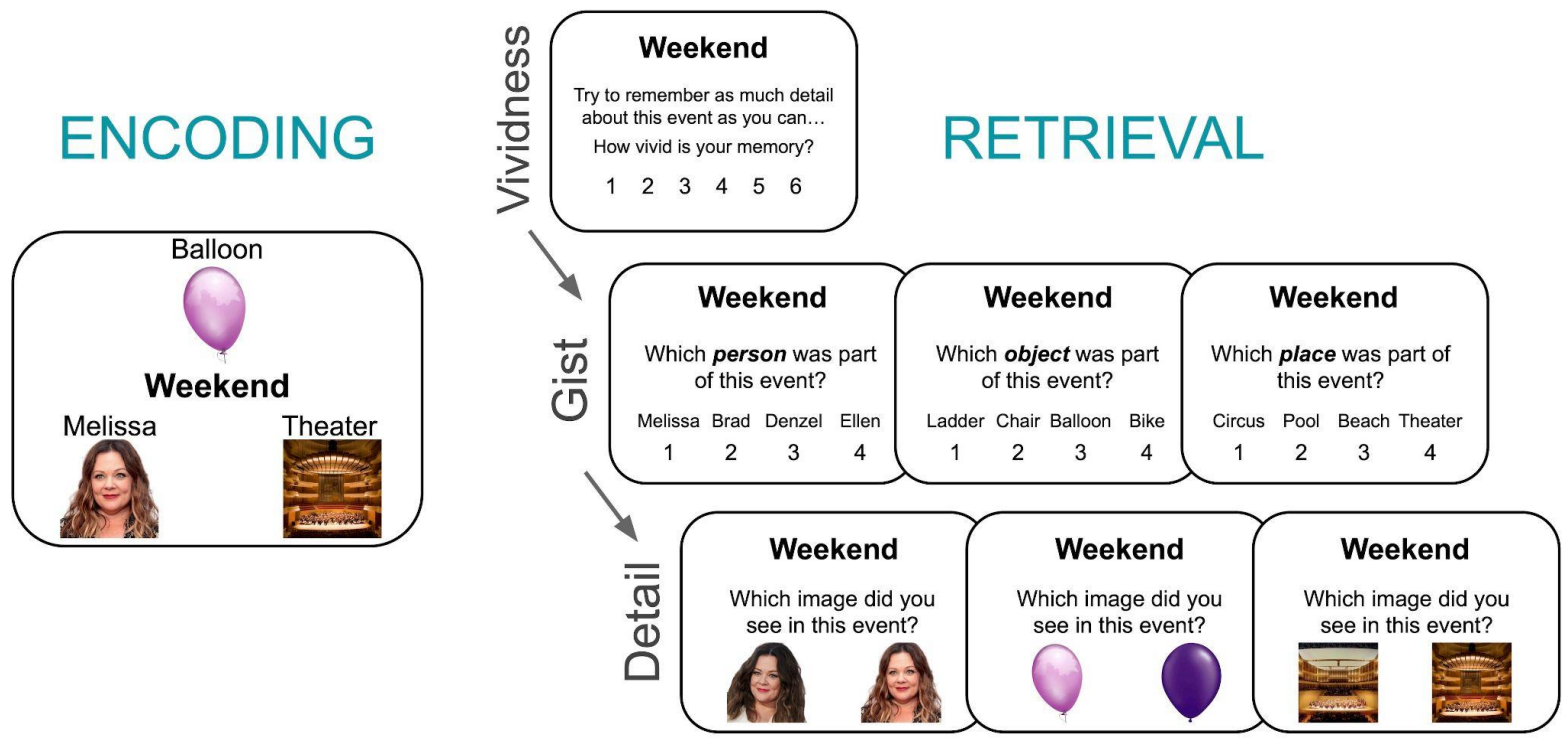

Fig 1. Task design. Example of a study phase trial (left: "Encoding"), where participants studied 3 elements (a person, place, and object with their labels) and a theme word (e.g., 'Weekend') and were asked to generate a story linking the 3 elements together around the theme word. On every trial in the memory test (right: "Retrieval"), participants saw a theme word as a memory cue and were asked to rate their subjective memory vividness indicating how well they could recall the entire event. Next, they were asked to identify the associated elements at the gist-level (a choice between 4 word labels). Finally, participants remembered each element at the detail-level (a choice between two perceptually similar images).

\section{Statistical Analyses}

For each participant, every event (a unique combination of a theme word, person, object, and place) was characterized by 7 memory measures: a vividness rating (1 to 6), as well as gist and detail memory ( 0 , incorrect, or 1 , correct) for the person, object, and place. The subjective vividness rating is regarded as the target measure for each event, with the objective measures of gist and detail memory for the event elements referred to hereafter as memory "attributes". The overarching goal of our analyses was to quantify the relationship between objective memory attributes and subjective memory vividness. We predicted that gist and detail memory would additively contribute to memory vividness, and that this relationship would be particularly strong for place information. All analyses were run in Python with JupyterLab.

First, we implemented several data quality checks to determine which participants would be included in the analyses. We excluded participants who were not paying attention to the task, defined by a median reaction time less than 0.75 s or pressing the same key more than $75 \%$ of the 
time. We additionally excluded participants whose memory performance was close to or below chance, using a threshold of less than $30 \%$ correct for gist memory (chance $=25 \%$ ) or less than $55 \%$ correct for detail memory (chance $=50 \%$ ). Finally, although not necessarily an indicator of poor task engagement, we additionally excluded participants who had responded with the same vividness rating more than $90 \%$ of the time. Given that our analyses of interest focused on trial-wise relationships with memory vividness, a lack of variance would prohibit or bias such analyses.

Two methods were used to assess the trial-wise relationship between vividness and memory attributes - within-participant correlations as well as predictive linear regression models. Note that, for these analyses, detail memory was marked as incorrect for trials where gist memory was also incorrect. This is because detail information (always tested for the correct association) cannot contribute to vividness judgments if the gist of that feature was not first correctly recalled. To assess how subjective vividness was related to memory attributes, we first verified that vividness ratings correlated with overall memory, i.e., the total number of correct memory attributes recalled (0-6) for each event. Number of attributes was correlated with vividness across trials within each participant using Spearman's correlation. Next, we separately investigated the influence of gist and detail memory for each element. For detail memory measures, trials were restricted to those where the gist was successfully recalled to test the additive influence of memory for perceptual detail over and above memory for gist. In all cases, participant-specific correlation coefficients were Fisher-z transformed before running analyses at the group level. Bonferroni-corrected one-sample t-tests were used to test if the correlation between memory attributes and vividness significantly differed from 0 . To test the differential effect of specificity and content, we further conducted a specificity (2 levels: gist, detail) x content (3 levels: object, person, place) repeated-measures ANOVA.

The second method employed a predictive model across all participants to test whether trial-wise memory attributes were able to predict vividness ratings of left-out events, and which conjunction of attributes explained the most variance in memory vividness. Using the scikit-learn package (Pedregosa, 2011), we built linear regression models tested with repeated stratified cross validation, using 10 folds repeated 5 times, for a total of 50 train-test splits. The full model included 6 predictors for the individual memory attributes. Note that, by including both gist and 
detail measures in the same model, gist now reduces to gist memory without correct detail memory, with detail predictors still testing the additive influence of detail memory on vividness. After fitting this full model, we inspected the mean beta weights of each predictor. The feature with the smallest weight was then removed from the model and the full cross validation procedure was run again with $\mathrm{N}-1$ predictors. This procedure was repeated until only 1 predictor remained. The mean out-of-sample R-squared value (variance explained in the test set vividness ratings) was stored for each model, and the best model was defined as that with the highest out-of-sample R-squared value.

\section{Results}

We first checked overall performance on the subjective and objective memory measures (see Table 1, which includes summary memory measures from all three experiments). In Experiment 1, participants were consistently able to remember the gist and detail of the elements associated with the theme word cues well above chance, but performance was not at ceiling, whereas vividness ratings were relatively low on average.

Table 1. Mean (and standard error) of memory scores for subjective memory vividness (1-6) and proportion correct for objective memory content (object, person, place) and specificity (gist - chance $=0.25$, detail chance $=0.5$ ) for all experiments.

\begin{tabular}{|c|c|c|c|c|c|c|c|}
\hline \multicolumn{2}{|c|}{} & \multicolumn{2}{|c|}{ Object } & \multicolumn{2}{c|}{ Person } & \multicolumn{2}{c|}{ Place } \\
\hline & Vividness & Gist & Detail & Gist & Detail & Gist & Detail \\
\hline $\begin{array}{c}\text { Experiment 1 } \\
(N=48)\end{array}$ & $2.62(0.14)$ & $0.71(0.03)$ & $0.76(0.02)$ & $0.66(0.03)$ & $0.74(0.02)$ & $0.73(0.03)$ & $0.71(0.02)$ \\
\hline $\begin{array}{c}\text { Experiment 2 } \\
(N=35)\end{array}$ & $3.51(0.16)$ & $0.83(0.03)$ & $0.76(0.02)$ & $0.77(0.02)$ & $0.77(0.02)$ & $0.84(0.03)$ & $0.73(0.02)$ \\
\hline $\begin{array}{c}\text { Experiment 3, } \\
\text { Person }(N=36)\end{array}$ & $3.96(0.17)$ & $0.82(0.03)$ & $0.79(0.02)$ & $0.74(0.03)$ & $0.77(0.02)$ & $0.82(0.03)$ & $0.74(0.02)$ \\
\hline $\begin{array}{c}\text { Experiment 3, } \\
\text { Place }(N=36)\end{array}$ & $3.78(0.17)$ & $0.80(0.03)$ & $0.73(0.02)$ & $0.71(0.03)$ & $0.75(0.02)$ & $0.79(0.03)$ & $0.73(0.02)$ \\
\hline
\end{tabular}

Next we moved to address our central question: How do objective memory attributes relate to subjective judgments of memory vividness? First, we verified that overall objective memory accuracy does in fact relate to subjective judgments of memory vividness by correlating the total 
number of attributes recalled (0-6) with vividness ratings across the 24 events within each participant. There was a significant correlation between trial-wise total correct memory attributes and vividness on average across participants (mean z $=0.24$, SE $=0.04, \mathrm{t}(47)=6.76, p<.001, d=$ 0.98). We then considered the change in memory vividness related to the accuracy of individual memory attributes, testing the correlations with both content (object, person, place) and specificity (gist, detail) with Bonferroni-corrected one-sample t-tests (see Figure 2a). In calculating the relationship between vividness and detail memory, trials were restricted to those where the gist had been correctly recalled to test whether there is an additive influence of detail memory on subjective vividness.

One-sample t-tests (corrected p-values reported) revealed that gist memory for all 3 event elements was significantly related to vividness ratings (mean $z>0.11$, ts $(47)>3.97, p s<.002, d>$ 0.57). However, only detail memory for the place was related to vividness (mean $z=0.11, t(47)=$ 3.13, $p=.018, d=0.45)$ - this relationship was not present for object and person detail memory (mean $z<0.02$, ts $(47)<0.41, p s=1, d<0.06$ ). To test whether there was a differential effect of content and specificity on vividness, we ran an additional repeated measures ANOVA. There was a robust effect of specificity, where vividness ratings were more strongly tied to successful retrieval of gist-level information than to memory for perceptual details $\left(F(1,47)=10.54, p<.001, \eta^{2}=\right.$ $0.07)$, but non-significant effects of content $\left(F(2,94)=2.96, p=.057, \eta^{2}=0.02\right)$ and the interaction between content and specificity $\left(\mathrm{F}(2,94)=2.25, p=.11, \eta^{2}=0.02\right)$. For completeness, we additionally computed the within-participant correlations between the 6 objective memory attributes (Figure 2c), with detail memory not conditional upon correct gist memory, which showed a tight coupling in gist memory among the three event elements, but no correlation in memory for their perceptual detail.

The previous analyses tested the relationship between individual memory attributes and subjective memory vividness, but did not account for their covariance and unique contributions, or their ability to predict vividness ratings of out-of-sample events. Therefore, as a complementary analysis, we next ran a series of predictive linear regression models across all trials, with the full model containing the 6 memory attributes. Note that, by including all measures within the model simultaneously, gist memory regressors now reflect gist memory in the absence of detail memory, and detail memory regressors reflect correct gist and detail memory. On each iteration, the model 
was simplified by removing the predictor that made the lowest contribution to memory vividness ratings. The beta coefficients of the full model are shown in Figure 2b. Objective memory attributes were able to predict the subjective vividness ratings of held-out trials, albeit explaining relatively low variance. The best model (highest out-of-sample R-squared $=.086$; $S D=.046$ ) did not include place gist memory or object detail memory, with place detail memory alone accounting for the most variance in vividness ratings ( $\mathrm{R}$-squared $=.056$; $\mathrm{SD}=.039)$. Therefore, in Experiment 1, we found a collective relationship between successful retrieval of gist-level information of objects, people, and places and subjective memory vividness, with relatively little unique variance explained by any feature alone. In contrast, there was a disproportionate, unique benefit of memory for detailed place information on vividness ratings, such that place information was predictive of vividness ratings but only if specific perceptual details of the place were additionally recalled.

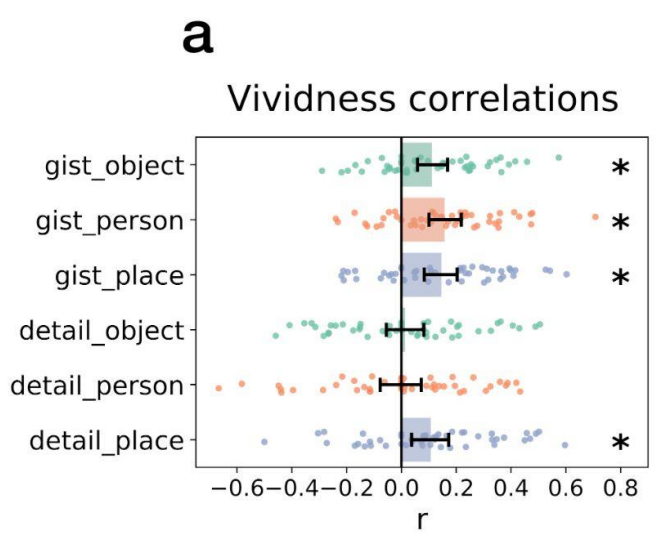

b

Full model feature weights

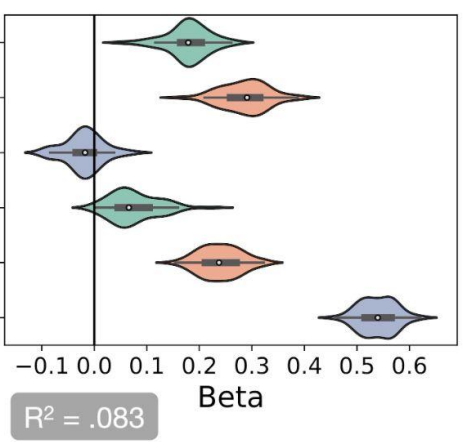

C

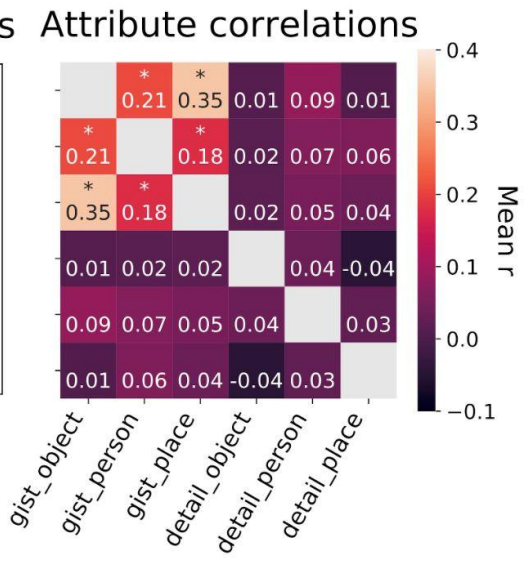

Fig 2. Experiment 1 results. a) Within-participant correlations between memory vividness ratings and memory attributes. ${ }^{*}$ indicates $p<.05$, Bonferroni-corrected. Detail correlations are restricted to trials where the gist was correct. Bars indicate the mean $+/-95 \%$ Cl. b) The beta coefficients of memory attributes included in the full predictive model. The violins show the range of beta values obtained across the 50 train-test splits. Out-of-sample R-squared of the full model is shown in the gray box. c) Within-participant correlations (group-averaged values plotted) between memory attributes. * indicates $p<.05$, Bonferroni-corrected across all unique correlations. 


\section{Experiment 2}

Experiment 1 revealed that subjective vividness was related to recalling the gist of all event elements as well as detailed place information, albeit somewhat weakly. In asking for vividness ratings at the beginning of each test trial, prompted by only the theme word cue, we found that vividness ratings were low on average $($ mean $=2.62 / 6)$ with a skewed right distribution (participants were approximately 5 times more likely to respond with a rating of 1 , which was the modal response, than a 6). We interpreted this pattern as indicating that the theme word alone provided little retrieval support for the event memory, possibly leading to a disconnect between our forced-choice measures of memory and the vividness ratings. We hypothesized that perceptual details of all elements might contribute more to vividness ratings if greater retrieval support was provided prior to making this subjective judgment. To address this issue, for Experiment 2, the vividness question was moved after the three gist memory questions, allowing participants to use these questions as additional memory cues and providing an opportunity for more vivid recall.

\section{Methods}

A total of 35 participants were included in analyses for Experiment 2. We recruited 32 participants from MTurk and 16 participants from Boston College. After data quality checks (as detailed in Experiment 1: Statistical Analyses), 13 participants were excluded (all MTurk), which was 27\% of the total sample, leaving a final sample size of 35 (19 male, 16 female, mean age $=28.1$ years, SD $=9.0, \min =18, \max =47)$. Sona participants made up $46 \%$ of the final sample. The smaller sample size for Experiment 2 was based on a power analysis from a main effect of interest in Experiment 1 - the mean within-participant correlation between vividness and gist memory with a Cohen's $d=0.71$.

The only change to the task design from Experiment 1 was the timing of the vividness question in Experiment 2, it was now asked immediately after the gist questions, not before. No feedback was given about the accuracy of their gist memory. Additionally, 3 face pairs were substituted for 3 new ones because less than two-thirds of participants in Experiment 1 indicated that they could recognize and name the people shown in those images. Otherwise, images remained identical to 
those used in Experiment 1, and the groupings of images and theme words into events were re-randomized.

\section{Results}

Experiment 2 increased retrieval support for vividness judgments by asking for this rating after the gist of each element had been tested. Based on this procedural difference, we expected vividness ratings to increase overall relative to Experiment 1. Verifying the effect of retrieval support, participants in Experiments 2 tended to report higher mean memory vividness ratings (mean = 3.51; see Table 1), with a more uniform distribution (the modal rating was now 4, with a similar probability of responding with a rating of 1 or 6$)$. Gist memory and detail memory remained well above chance, although memory for gist appeared to increase after Experiment 1. Because event elements and theme words were recombined into new events for Experiment 2, it is possible that the new events were easier to create stories for (and, thus, easier to associate the elements with the theme and each other). Consistent with this interpretation, on average, participants were 1 second faster to generate stories at encoding in Experiment 2 (mean $=8.17 \mathrm{~s}, \mathrm{SE}=0.40$ ) compared to Experiment 1 (mean $=9.09 \mathrm{~s}, \mathrm{SE}=0.35$ ).

Turning to the relationship between memory vividness and memory attributes, we found a strong relationship on average between the total number of correct memory attributes per trial and vividness ratings (mean $\mathrm{z}=0.58, \mathrm{SE}=0.04, \mathrm{t}(34)=14.05, p<.001, d=2.37$ ). When analyzing the correlation between vividness and each of the 6 attributes separately (see Figure 3a), we replicated the overall pattern from Experiment 1: Gist memory for all 3 kinds of content was significantly related to vividness ratings (mean $z>0.33$, ts $(34)>7.79$, ps $<.001, d>1.31$ ), and only detail memory for the place was significantly related to vividness (mean $z=0.17, t(34)=$ 3.08, $p=.025, d=0.52)-$ this relationship was not significant for object and person detail memory (mean $z<0.10$, ts $(34)<2.44$, $p s>.12, d<0.42$ ). A repeated measures ANOVA showed that vividness ratings were more strongly tied to successful retrieval of gist-level information compared to memory for perceptual details $\left(F(1,34)=51.25, p<.001, \eta^{2}=0.28\right)$, but there were no differences based on content $\left(\mathrm{F}(2,68)=0.65, p=.52, \eta^{2}<0.01\right)$. However, the interaction between content and specificity was significant $\left(F(2,68)=5.49, p=.006, \eta^{2}=0.04\right)$, which appeared to be driven by a stronger relationship between vividness and person gist memory compared to person detail memory. Finally, predictive linear models were run using the same 
method as described in Experiment 1 (see Figure 3b). The best model for Experiment 2 $(\mathrm{R}$-squared $=.303 ; \mathrm{SD}=.079)$ dropped only person detail memory, and the overall variance explained in vividness ratings was much higher than in Experiment 1, with unique variance now explained by each gist memory measure. Additionally, the dominant effect of place detail memory found in Experiment 1 was no longer observed. Therefore, when more retrieval support was provided by assessing vividness after the gist memory test, each gist memory attribute, in particular, contributed independently and more strongly to vividness ratings.

\section{a}

Vividness correlations

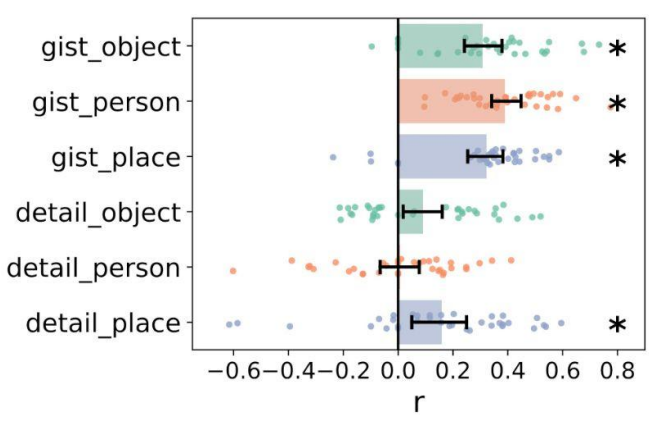

b

Full model feature weights

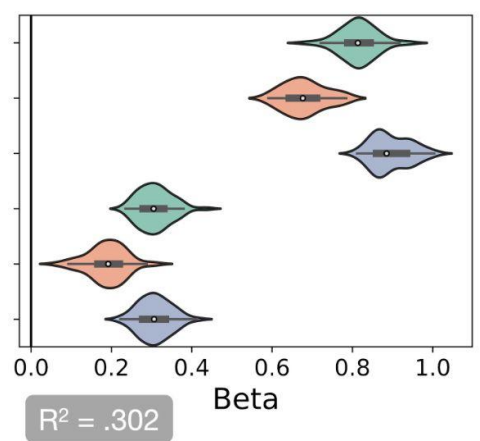

C

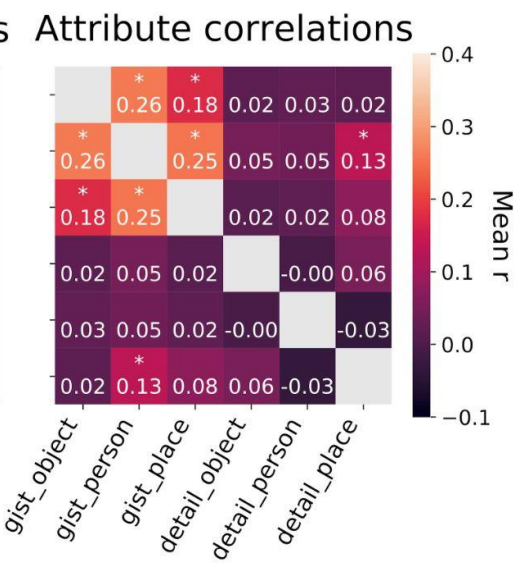

Fig 3. Experiment 2 results. a) Within-participant correlations between memory vividness ratings and memory attributes. ${ }^{*}$ indicates $p<.05$, Bonferroni-corrected.; bars indicate the mean $+/-95 \% \mathrm{Cl}$. b) The beta coefficients of memory attributes included in the full predictive model across the 50 train-test splits. Out-of-sample R-squared of the full model is shown in the gray box. c) Group-averaged within-participant correlations between memory attributes. ${ }^{*}$ indicates $p<.05$, Bonferroni-corrected.

\section{Experiment 3}

By providing retrieval support for vividness judgments, Experiment 2 boosted the relationship between gist memory, in particular, for each of the event elements and memory vividness, but detail memory still had an additive, yet smaller influence. We next sought to test whether the relationship between memory content and vividness could be modulated by attentional focus at encoding. In Experiment 3, we directed participants to prioritize either the person or the place when encoding each event. We predicted that vividness judgments would show a flexible relationship with memory content, changing as a function of encoding orientation, such that 
person memory would relate to vividness more strongly if preferentially encoded, and similarly for place information.

\section{Methods}

A total of 72 participants were included in analyses for Experiment 3. 80 participants were recruited, all from the Boston College Sona community after confirming that there were no systematic differences in the pattern of memory effects between Sona and MTurk participants in the first two experiments. After data quality checks (as detailed in Experiment 1: Statistical Analyses), 8 participants were excluded. Experiment 3 employed a between-subjects design, with a final sample size of 36 participants per group (Group A: 11 male, mean age $=19.53$ years, SD = 1.32, $\min =18, \max =24 ;$ Group B: 11 male, mean age $=19.66$ years, $S D=1.24, \min =18, \max =$ 22). Age did not significantly differ between groups $(p=.67)$.

The task design for Experiment 3 was the same as Experiment 2, but with the addition of a between-subjects encoding manipulation. Participants were instructed to prioritize either the person (Group A) or the place (Group B) within the event, with the prioritized element highlighted with a blue square during the study phase, and to frame the story around that element. In order to check whether there were differences in how well participants could frame a story around the person or place, we also introduced a success rating after each encoding trial, where participants reported how successful they were in generating a story on a scale of 1 to 6 .

\section{Results}

We expected that focusing on the person would disproportionately enhance gist and detail memory for this element, whereas focusing on the place feature would disproportionately enhance memory for the scene. Surprisingly, instructions to focus on the person or place had no significant effect on the ability to remember those elements. When comparing gist memory between groups (person vs. place encoding), there was no main effect of group $\left(F(1,70)=0.42, p=.52, \eta^{2}<0.01\right)$ nor interaction between group and element type $\left(\mathrm{F}(2,140)=0.37, p=.69, \eta^{2}<0.01\right)$. The same was true for detail memory (main effect of group: $\mathrm{F}(1,70)=1.53, p=.22, \eta^{2}=0.01$; interaction: $\left.\mathrm{F}(2,140)=1.62, p=.20, \eta^{2}=0.01\right)$. There were also no significant group differences in mean memory vividness ratings $(\mathrm{t}(70)=0.76, p=.45, d=0.18)$ or the ease with which stories could be generated at encoding $(\mathrm{t}(70)=0.94, p=.35, d=0.22)$. Thus, we concluded that our encoding 
manipulation had little effect on how participants remembered the events. As a result, data from Experiment 3 were collapsed across groups for further replication analyses (conducted in the same way as Experiments 1 and 2).

Replicating the prior experiments, there was a strong relationship between correct memory attributes and memory vividness (mean $z=0.59$, SE $=0.04, \mathrm{t}(71)=14.94, p<.001, d=1.76$ ). However, with this larger sample, we now observed significant relationships between vividness and all 6 of the individual attributes (see Figure 4a) (mean $z>0.09$, ts $(71)>3.02, p s<.022, d>$ 0.39). Running a repeated measures ANOVA revealed a strong effect of specificity $(F(1,71)=$ 97.94, $p<.001, \eta^{2}=0.29$ ), but no effect of memory content (main effect: $F(2,142)=0.25, p=.78$, $\eta^{2}<0.01$; interaction: $\left.\mathrm{F}(2,142)=0.35, p=.70, \eta^{2}<0.01\right)$. In line with these results, the best fitting predictive linear model (see Figure 4b) of vividness was the full model, including all 6 memory attributes $(\mathrm{R}$-squared $=.414 ; \mathrm{SD}=.053)$.

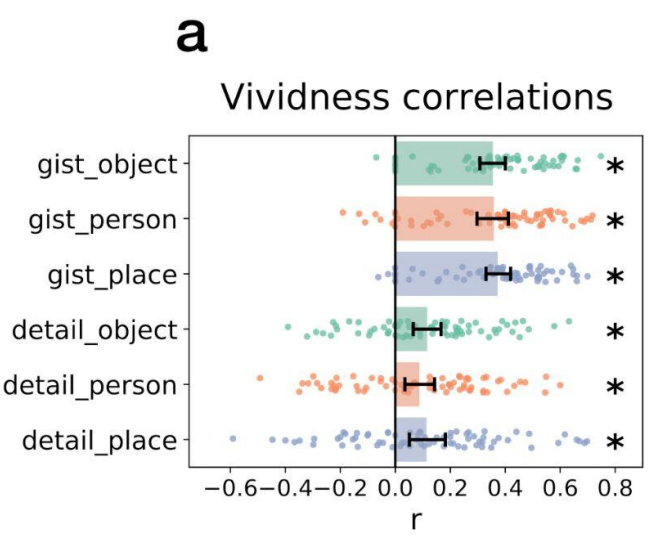

b

Full model feature weights

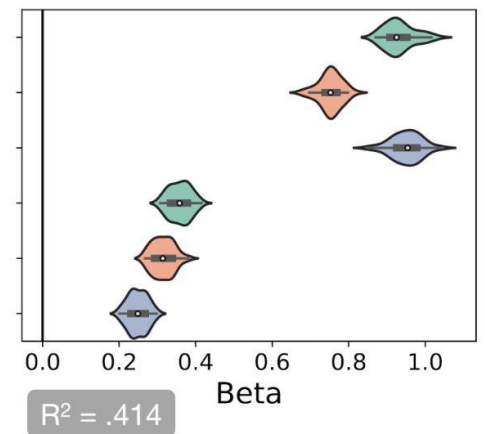

C

Attribute correlations

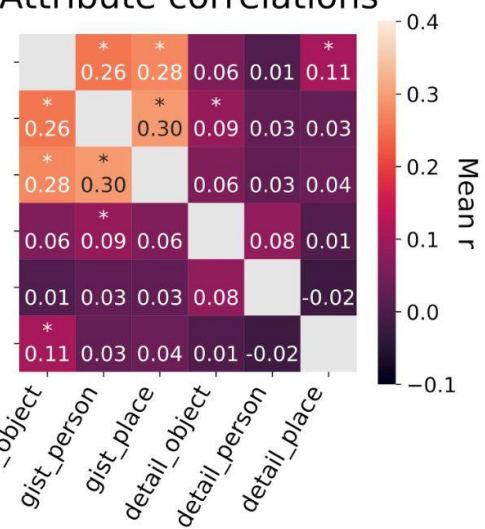

Fig 4. Experiment 3 results. a) Within-participant correlations between memory vividness ratings and memory attributes. ${ }^{*}$ indicates $p<.05$, Bonferroni-corrected.; bars indicate the mean $+/-95 \% \mathrm{Cl}$. b) The beta coefficients of memory attributes in the full predictive model across the 50 train-test splits. Out-of-sample R-squared of the full model is shown in the gray box. c) Group-averaged within-participant correlations between memory attributes. ${ }^{*}$ indicates $p<.05$, Bonferroni-corrected. 


\section{Individual Differences}

\section{Methods}

In addition to the trial-wise analyses performed within each experiment, we additionally performed two individual differences analyses across all participants from our three experiments. Prior to combining participants, memory scores were first z-scored within each experiment group and measure to remove any influence of differences in mean performance. Outliers were then removed who had any z-scored memory score with an absolute value greater than 2.5 . The first analysis simply correlated the mean scores for memory vividness with each of the 6 memory attribute measures across participants. We additionally assessed the relationship between all of our task memory measures and two additional subjective measures of memory and imagery from separate questionnaires - the SAM episodic scores (self-reported episodic memory ability) and visual imagery scores (strength of imagery from the vividness of visual imagery questionnaire; VVIQ).

The second analysis investigated whether the within-participant correlations between memory attributes and vividness (as analyzed within each experiment) tend to be consistent in their pattern across individuals. Do the same kinds of memory attributes tend to correlate with vividness across participants, with individual variability explained by the magnitude with which objective memory accuracy tracks subjective memory vividness, or do different participants base their vividness judgments on different kinds of memory attributes? Here, we ran a K-Means clustering analysis to group participants based on the similarity (Euclidean distance) of the correlations between the 6 memory attributes and memory vividness. The optimal number of clusters $(k)$ was determined by identifying the elbow of the curve of inertia values, defined as the sum of squared distances of each participant to their closest cluster center, calculated using between 1 and 10 clusters. For each $\mathrm{k}$ iteration, K-Means was run 500 times, returning the cluster assignments with the lowest inertia.

\section{Results}

\section{Correlations between memory measures}

After excluding outliers, a total of 147 participants were included in the across-participant correlation analyses (see Figure 5). Correlation coefficients were evaluated at a one-tailed 
(predicted positive correlation) Bonferroni-corrected threshold of $r=.232$. We expected that mean memory vividness scores from our experimental task should correlate with episodic memory and imagery ability from the questionnaires, given that all reflect subjective assessments of memory and imagination of visuo-spatial events. Both questionnaire measures were indeed significantly correlated with mean memory vividness, albeit weakly, across subjects ( $r s>.25$ ). Turning to the correlation with objective memory attributes, subjective vividness was significantly correlated with all 6 measures, showing a stronger relationship with gist memory than detail memory, consistent with the within-participant, trial-wise analyses. Correlation coefficients were similar in magnitude for all types of memory content. Interestingly, subjective episodic memory (SAM) scores and visual imagery scores were not significantly correlated with any event memory attributes apart from place detail memory, which showed a small positive correlation with SAM episodic memory $(r=.24)$.

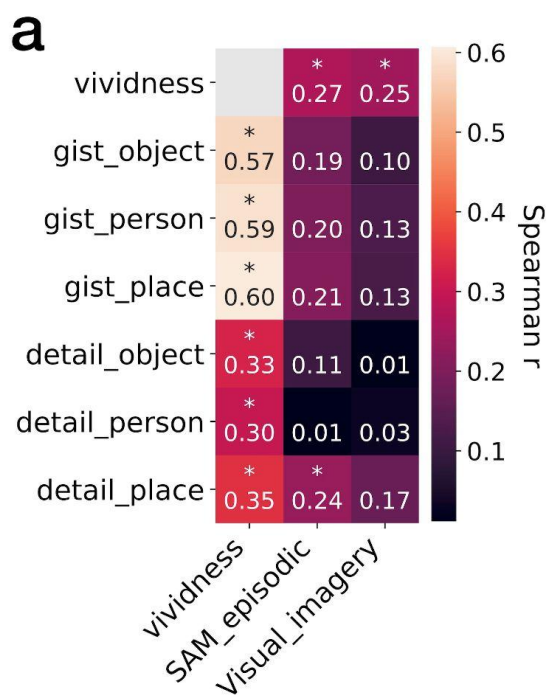

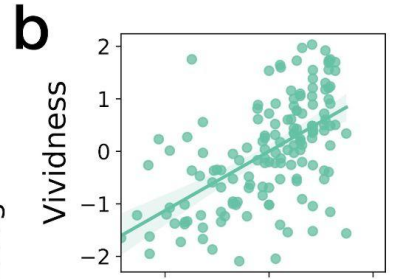

Gist Object

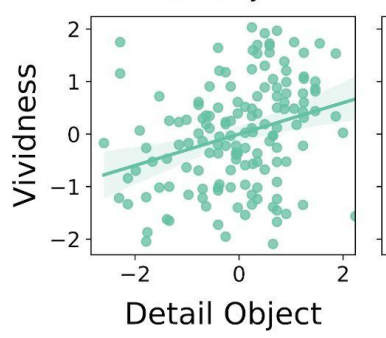

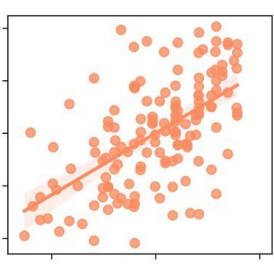

Gist Person

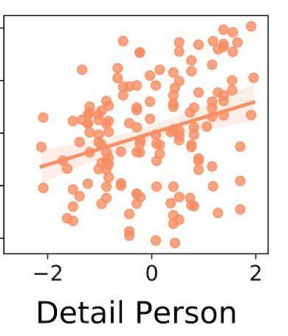

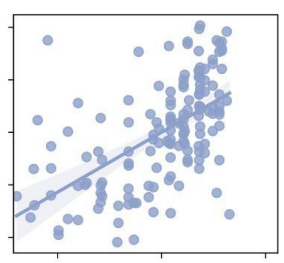

Gist Place

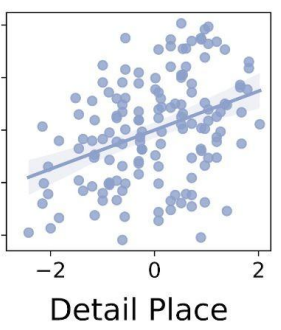

Fig 5. Correlations between memory measures across participants. a) Spearman $r$ correlation coefficients between each measure. * indicates $p<.05$ Bonferroni-corrected. b) Scatter plots illustrating the relationship between memory vividness and the six memory attributes (corresponding to the correlations shown in the first column of panel a).

\section{Clusters of vividness correlation patterns}

So far, the results have pointed to a strong relationship between gist memory and subjective memory vividness, with evidence of a smaller additive influence of detail memory. However, assessing the dominant or average relationships across participants does not reveal if there is 
individual variability in the relationship between memory attributes and subjective vividness - do individuals vary not only in how strongly related objective and subjective memory measures are, but which memory attributes support vividness judgments? To answer this question, we ran a K-Means clustering analysis, which grouped subjects based on their patterns of trial-wise correlations between memory vividness and the six memory attributes.

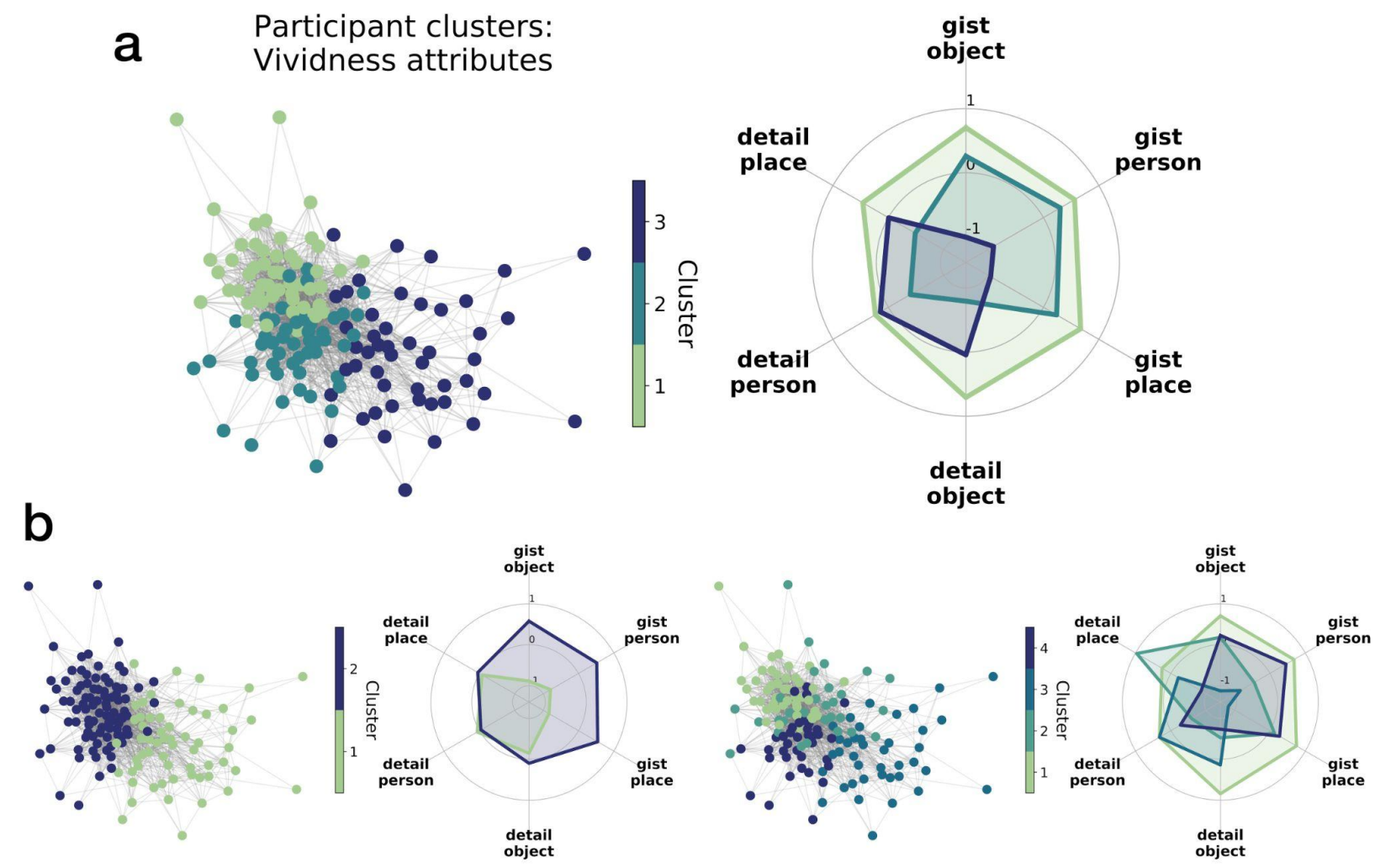

Fig 6. Memory profiles, showing clusters of participants based on similarity in attribute-to-vividness correlation patterns. a) Results from the 3-cluster (optimal) K-Means solution. Left: the euclidean distance between each subject (node) based on their attribute-to-vividness correlations, showing the top 20\% of edges for visualization and with participants color-coded by cluster assignment. Right: A polar plot showing the cluster centroid values (z scores) of the relationship between memory vividness and the six memory attributes. b) Results from the 2-cluster solution and the 4-cluster solution.

The optimal number of clusters for explaining inter-subject variability in vividness correlations was 3 , and the clusters are shown in Figure 6a. The first cluster $(N=51)$ had the highest correspondence between memory vividness and memory attributes overall, so a generally tighter coupling between subjective and objective memory measures. Interestingly, the remaining two clusters separated by specificity: participants in the second cluster $(\mathrm{N}=48)$ showed a stronger 
relationship between gist memory and vividness, whereas participants in the third cluster $(\mathrm{N}=48)$ showed a relatively stronger relationship between detail memory and vividness. Confirming the results of our cluster separation, between-subject ANOVAs indicated a significant difference between the clusters for all attributes and their relationship to vividness (Fs $(2,144)>6.01$, ps < $\left..004, \eta^{2}>0.07\right)$. For completeness, we additionally plotted the results of the 2 - and 4-cluster K-Means analysis (Figure 6b). The 2-cluster solution verified a primary division based on magnitude and also specificity, and the 4-cluster solution pointed to an additional separation based on content, where the vividness ratings of one cluster of participants were correlated particularly strongly with perceptually detailed place information.

A follow-up, control analysis on the three clusters confirmed that they were not predicted by experiment group $\left.\chi^{2}(6)=3.70, p=.72\right)$. Moreover, the clusters did not significantly differ in performance on any of the memory task measures $\left(\mathrm{Fs}(2,144)<1.19\right.$, ps $\left.>.30, \eta^{2}<0.02\right)$ or on the SAM and VVIQ questionnaires (Fs $(2,144)<2.05$, ps $\left.>.13, \eta^{2}<0.03\right)$, meaning that the relationship between memory attributes and vividness was not significantly modulated by measured or perceived memory ability. Therefore, participants do not vary only in how strongly objective memory accuracy in general tracks with memory vividness (Cluster 1 vs. 2 and 3), but also in how tightly coupled memory for gist-level information (Cluster 2) and specific perceptual details (Cluster 3 ) are to this subjective judgment.

\section{General Discussion}

Over three experiments, we investigated the relationship between attributes of event memories, including their content and specificity, and subjective evaluations of memory vividness. We observed a reliably strong correspondence between memory for gist information (names of objects, people, and places) and vividness ratings. Memory for specific perceptual details of correctly identified event elements provided an additive, yet smaller, boost to memory vividness, and, in some cases, this was particularly apparent for place information. Although this was the dominant pattern across participants, we further used a data-driven clustering approach to reveal individual differences in how memory attributes support subjective vividness, with some participants relying much more on gist-level information, and others drawing upon their memory for perceptually detailed information. Overall, these findings support the importance of an 
integrated, gist-level framework for episodic recollection and the additive, independent benefit of remembering specific perceptual details on memory vividness. They also provide some support for the primacy of detailed place information in memory, although this result was not consistent across experiments. Importantly, however, we further highlight idiosyncrasies in the relationship between objective memory attributes and evaluations of memory vividness, primarily in relation to memory specificity, raising the broader question of to what extent, and in which pattern, these measures converge.

A dominant characteristic of episodic memory is holistic retrieval, defined as the dependent, relational structure of event elements. This means that when the gist of one element is recalled, you are much more likely to be able to recall the other associated elements than by chance (if the elements were represented independently) (Horner \& Burgess, 2013). Across all experiments we found evidence that, at the trial level, gist memory was a robust determinant of memory vividness, which was also supported by strong correlations between mean vividness and gist memory across participants. We additionally found that gist memory across trials was strongly correlated between the different kinds of content. These findings support the idea that event elements remembered at a gist level of specificity are dependent in memory (Joensen et al., 2020; Ngo et al., 2020), informing vividness judgments as a bound representation. Furthermore, in Experiments 2 and 3, with gist information more readily available when making vividness judgments, we found that recalling the gist alone (even with incorrect detail memory) was a strong predictor of vividness ratings. Therefore, having an episodic memory that is more conceptual in nature, but that still contains a lot of information, may be sufficient for subjectively vivid reexperiencing, at least in some individuals.

At the same time, we found that memory for perceptual details of event elements boosted memory vividness independently but to a lesser extent, showing a smaller correlation at the both trial and individual level. Moreover, in contrast to gist information, we found that detail memory was not correlated across the different event elements, which supports our prior work showing an independence of different kinds of perceptual detail in memory (Cooper \& Ritchey, 2019). However, the additive influence of detail memory on vividness does support evidence of a correspondence between low level visual characteristics of scenes and vividness ratings (Bone et al., 2020; Cooper et al., 2019). One challenge in analyzing the relative correlation of gist and detail 
memory with vividness is the different chance levels of these questions in our paradigm ( $25 \%$ and $50 \%$, respectively), leading to potentially greater noise in the detail memory trials. This potential discrepancy, however, cannot fully explain relative changes in the strength of the relationship between gist memory and vividness across experiments. This relationship was disproportionately stronger when gist-level associations were made more readily accessible in Experiments 2 and 3 compared to Experiment 1, further underscoring the dependency between gist memory accuracy and subjective vividness ratings.

In contrast to gist memory, relationships between detail memory and vividness also appeared more sensitive to content-level differences. In Experiment 1, detail memory for place information, specifically, was the strongest predictor of vividness ratings. This was seen to a lesser extent in Experiment 2, and was not replicated in Experiment 3, where detail memory for all types of event elements also enhanced memory vividness. We speculate that the dominant effect of place detail memory in Experiment 1, which was particularly apparent in the predictive model by accounting for the most unique variance in vividness, reflects the automatic saliency of place information in memory (Robin et al., 2018) when retrieval support is low. Interestingly, our individual differences analyses across all experiments showed that detailed place memory was the only objective memory measure to significantly correlate with independent ratings of subjective episodic memory ability (from the SAM questionnaire), further suggesting that the imageability of past events is often tied to the retrieval of a perceptually precise scene. The decrease in the importance of place detail information and the increased relevance of gist memory for subjective vividness after Experiment 1 are surprising and notable findings after changing only the timing of the vividness question. We predicted that asking the question after gist memory might increase the accessibility of perceptual detail of the event elements when making vividness judgments. It is possible, however, that participants were using specific perceptual characteristics of the event elements (such as colors and shapes) when making vividness ratings, but that this was not captured well by our lure discrimination task, where targets and lures share some but not all specific perceptual details. Future research will be needed to fully explore the conditions under which perceptual detail, and place information specifically, is tied to subjective evaluations of memory.

The current study measured vividness in the context of memory, but it is an open question whether the pattern of relationships that we observed here generalizes to other kinds of internal 
event representations, such as imagining fictitious scenarios or possible future ones. Imagined events are generally less perceptually vivid than remembered events (Addis et al., 2009; Berntsen \& Bohn, 2010), with future events placing greater demand on constructive processes, but are vividness judgments informed in the same way across these different tasks? Mental imagery is a core component of vividness judgments for both episodic memory and future thinking and, thus, direct insight into the foundations of vividness can be provided by studying people with aphantasia, characterized by a lack of mental imagery (for a review, see Pearson, 2019). In a recent study, Bainbridge and colleagues asked aphantasic and control participants to draw scenes they had studied from memory, finding that aphantasic participants drew fewer objects, with fewer specific details and colors, but were able to accurately remember their overall spatial layout (Bainbridge et al., 2020). This work is supported by a detailed self-report profile of memory and imagery in aphantasia, showing impaired imagery of episodic memories, objects, and fictitious events, but intact spatial navigation and spatial imagery (Dawes et al., 2020). These findings from aphantasia align with research showing a tighter coupling between self-reported object imagery (perceptual details of scene components) and autobiographical memory, compared to imagery of schematic spatial relations (Fan et al., 2020). One interpretation is that spatial imagery facilitates the retrieval of event context whereas object imagery supports reflective evaluations such as vividness (Aydin, 2018). Together, research in this field suggests that, while representation of broad contextual details of an event are likely necessary for a coherent mental representation of an event, the experience of vividness is further driven by memory for specific perceptual details within that event, lending support to the additive influence of detail memory on vividness judgments that we observed.

Although group-level analyses paint a certain picture about the objective basis of vividness ratings, it is important to account for and explain individual differences in how people judge their experience of remembering. Reported memory vividness is thought to be stable within individuals and has been referred to as more of a trait rather than state-based phenomenon (Berg et al., 2021). Yet it is unclear if people tend to base their vividness judgments on the same memory attributes. Using a clustering analysis, we found that participants differed in which memory attributes related to their judgments of memory vividness, and this variation was largely accounted for by memory specificity: some individuals reported a vivid memory as long as they had retrieved gist-level associations, whereas a vivid memory for others was tied to memory for perceptual 
detail of retrieved elements. Analogous findings have been observed in the context of age-related memory changes. Older adults tend to report just as high if not higher ratings of memory vividness than younger adults, but there is a disconnect between subjective memory and specific episodic memory details (Folville, Simons, et al., 2020) at both the individual (Duarte et al., 2008) and trial level (Folville, D’Argembeau, et al., 2020). One explanation is that older adults weight semantic or internal information within the prefrontal cortex more strongly when making vividness ratings in contrast to younger adults, who may rely more on the reactivation of perceptual detail in the posterior parietal cortex (Johnson et al., 2015). Research has suggested that reactivation of semantic, gist information is dependent upon hippocampal interactions with an anterior, prefrontal brain network, whereas reactivation of perceptual detail involves interactions with a posterior medial network (Robin \& Moscovitch, 2017; Sheldon et al., 2016; Sheldon \& Levine, 2018). Therefore, it is possible that individual and population differences in the contributions of gist and detail memory to subjective vividness relate to biases in hippocampal interactions with anterior and posterior brain regions, respectively. Of note, it remains unclear from our analyses and task design whether gist and detail information emerge from distinct semantic and perceptual representations or from one continuum of representational specificity (cf. Schurgin et al., 2020). Regardless though, our results highlight that there are individual differences in how coarse and precise memory representations relate to subjective memory vividness.

One limitation of our experimental task is that there are many other attributes of event memories, beyond the elements tested here, that may be important drivers of vividness and subject to individual and population differences, including internal thoughts and affective states. Even in Experiments 2 and 3 where objective memory attributes and subjective vividness were more tightly coupled across trials within participants, the mean within-participant correlation was relatively low (approximately $0.1-0.4$ ), with a moderate correlation across participants (approximately $0.3-0.6$ ), suggesting a substantial amount of variance in subjective vividness can be explained by other factors. For example, emotional memories, particularly unpleasant experiences, are re-experienced especially vividly (see Kensinger \& Ford, 2020), and are characterized by a prioritization of sensory information over semantic, contextual information (Bowen et al., 2018). Interestingly, though, vividness judgments of negative memories may be less strongly tied to memory for perceptual details compared to non-emotional memories (Cooper et al., 2019), suggesting that vividness is additionally influenced by the affective significance of the 
remembered event. In fact, internal vividness (based on memory for internal states), and external vividness (based on memory for perceptual information), have been regarded as separate cognitive and neural processes (Ford \& Kensinger, 2016), but it is an open question how they are weighted in an overall, subjective evaluation of memory. Another key component of events is social dynamics, or any component that unfolds over time. Accounting for the influence of social content on evaluations of memory vividness, and individual differences in the prioritization of social information within events, is an important question for future research to address. Relatedly, we aimed to directly manipulate the prioritization of social (faces) versus non-social (places) in our final experiment, but were unable to validate the encoding manipulation, which did not influence memory performance. The pattern of relationships between vividness and socio-affective memory measures could also provide a new window into metacognition and subjective experience in populations that exhibit differences in how information is encoded and represented in episodic memory, for example autism (Cooper \& Simons, 2019) and depression (Söderlund et al., 2014).

In conclusion, prior behavioral and neuroimaging research has widely utilized vividness ratings as a measure of memory and imagery, with less investigation of the relationship between objective and subjective measures. To our knowledge, there has not been a systematic exploration of the pattern with which different kinds of objective memory attributes predict vividness ratings to date. Testing memory for distinct event elements at two levels of specificity, we observed a robust relationship between the number of gist-level event elements recalled and memory vividness, and a smaller unique benefit of recalling specific perceptual details of those elements. However, this average pattern was, importantly, accompanied by separate subgroups of participants who differentially prioritized gist and perceptual details when judging how vividly they could remember an event. Investigating how the diverse attributes of event memories contribute to the subjective experience of recollection provides a fruitful new direction for understanding the pattern of memory and imagination differences across populations. 


\section{References}

Addis, D. R., Pan, L., Vu, M.-A., Laiser, N., \& Schacter, D. L. (2009). Constructive episodic simulation of the future and the past: distinct subsystems of a core brain network mediate imagining and remembering. Neuropsychologia, 47(11), 2222-2238.

Aydin, C. (2018). The differential contributions of visual imagery constructs on autobiographical thinking. Memory , 26(2), 189-200.

Bainbridge, W. A., Hall, E. H., \& Baker, C. I. (2019). Drawings of real-world scenes during free recall reveal detailed object and spatial information in memory. Nature Communications, 10(1), 5.

Bainbridge, W. A., Pounder, Z., Eardley, A. F., \& Baker, C. I. (2020). Quantifying Aphantasia through drawing: Those without visual imagery show deficits in object but not spatial memory. Cortex; a Journal Devoted to the Study of the Nervous System and Behavior. https://doi.org/10.1016/j.cortex.2020.11.014

Berg, J. J., Gilmore, A. W., Shaffer, R. A., \& McDermott, K. B. (2021). The stability of visual perspective and vividness during mental time travel. Consciousness and Cognition, 92, 103116.

Berntsen, D., \& Bohn, A. (2010). Remembering and forecasting: The relation between autobiographical memory and episodic future thinking. Memory \& Cognition, 38(3), 265-278.

Bone, M. B., Ahmad, F., \& Buchsbaum, B. R. (2020). Feature-specific neural reactivation during episodic memory. Nature Communications, 11(1), 1945.

Bonnici, H. M., Richter, F. R., Yazar, Y., \& Simons, J. S. (2016). Multimodal Feature Integration in the Angular Gyrus during Episodic and Semantic Retrieval. The Journal of Neuroscience: The Official Journal of the Society for Neuroscience, 36(20), 5462-5471.

Bowen, H. J., Kark, S. M., \& Kensinger, E. A. (2018). NEVER forget: negative emotional valence enhances recapitulation. Psychonomic Bulletin \& Review, 25(3), 870-891.

Brady, T. F., Konkle, T., Alvarez, G. A., \& Oliva, A. (2008). Visual long-term memory has a massive storage capacity for object details. Proceedings of the National Academy of Sciences of the United States of America, 105(38), 14325-14329.

Clark, I. A., \& Maguire, E. A. (2020). Do questionnaires reflect their purported cognitive functions? Cognition, 195, 104114. 
Cooper, R. A., Kensinger, E. A., \& Ritchey, M. (2019). Memories Fade: The Relationship Between Memory Vividness and Remembered Visual Salience. Psychological Science, 30(5), 657-668.

Cooper, R. A., Plaisted-Grant, K. C., Baron-Cohen, S., \& Simons, J. S. (2017). Eye movements reveal a dissociation between memory encoding and retrieval in adults with autism. Cognition, 159, 127-138.

Cooper, R. A., \& Ritchey, M. (2019). Cortico-hippocampal network connections support the multidimensional quality of episodic memory. eLife, 8. https://doi.org/10.7554/eLife.45591

Cooper, R. A., \& Simons, J. S. (2019). Exploring the neurocognitive basis of episodic recollection in autism. Psychonomic Bulletin \& Review, 26(1), 163-181.

Dawes, A. J., Keogh, R., Andrillon, T., \& Pearson, J. (2020). A cognitive profile of multi-sensory imagery, memory and dreaming in aphantasia. Scientific Reports, 10(1), 10022.

Dijkstra, N., Bosch, S. E., \& van Gerven, M. A. J. (2017). Vividness of Visual Imagery Depends on the Neural Overlap with Perception in Visual Areas. The Journal of Neuroscience: The Official Journal of the Society for Neuroscience, 37(5), 1367-1373.

Duarte, A., Henson, R. N., \& Graham, K. S. (2008). The effects of aging on the neural correlates of subjective and objective recollection. Cerebral Cortex , 18(9), 2169-2180.

Fan, C. L., Abdi, H., \& Levine, B. (2020). On the relationship between trait autobiographical episodic memory and spatial navigation. Memory \& Cognition. https://doi.org/10.3758/s13421-020-01093-7

Folville, A., D’Argembeau, A., \& Bastin, C. (2020). Deciphering the relationship between objective and subjective aspects of recollection in healthy aging. Memory , 28(3), 362-373.

Folville, A., Simons, J., D’Argembeau, A., \& Bastin, C. (2020). I remember it like it was yesterday: Age-related changes in the subjective experience of remembering. https://doi.org/10.31234/osf.io/5kgha

Ford, J. H., \& Kensinger, E. A. (2016). Effects of internal and external vividness on hippocampal connectivity during memory retrieval. Neurobiology of Learning and Memory, 134 Pt A, 78-90.

Geib, B. R., Stanley, M. L., Wing, E. A., Laurienti, P. J., \& Cabeza, R. (2017). Hippocampal Contributions to the Large-Scale Episodic Memory Network Predict Vivid Visual Memories. Cerebral Cortex , 27(1), 680-693.

Hassabis, D., \& Maguire, E. A. (2007). Deconstructing episodic memory with construction. Trends in Cognitive Sciences, 11(7), 299-306. 
Herweg, N. A., Sharan, A. D., Sperling, M. R., Brandt, A., Schulze-Bonhage, A., \& Kahana, M. J. (2020). Reactivated Spatial Context Guides Episodic Recall. The Journal of Neuroscience: The Official Journal of the Society for Neuroscience, 40(10), 2119-2128.

Horner, A. J., Bisby, J. A., Wang, A., Bogus, K., \& Burgess, N. (2016). The role of spatial boundaries in shaping long-term event representations. Cognition, 154, 151-164.

Horner, A. J., \& Burgess, N. (2013). The associative structure of memory for multi-element events. Journal of Experimental Psychology. General, 142(4), 1370-1383.

Joensen, B. H., Gaskell, M. G., \& Horner, A. J. (2020). United we fall: All-or-none forgetting of complex episodic events. Journal of Experimental Psychology. General, 149(2), 230-248.

Johnson, M. K., Kuhl, B. A., Mitchell, K. J., Ankudowich, E., \& Durbin, K. A. (2015). Age-related differences in the neural basis of the subjective vividness of memories: evidence from multivoxel pattern classification. Cognitive, Affective \& Behavioral Neuroscience, 15(3), 644-661.

Kensinger, E. A., \& Ford, J. H. (2020). Retrieval of Emotional Events from Memory. Annual Review of Psychology, 71, 251-272.

Kirwan, C. B., Jones, C. K., Miller, M. I., \& Stark, C. E. L. (2007). High-resolution fMRI investigation of the medial temporal lobe. Human Brain Mapping, 28(10), 959-966.

Kuhl, B. A., \& Chun, M. M. (2014). Successful remembering elicits event-specific activity patterns in lateral parietal cortex. The Journal of Neuroscience: The Official Journal of the Society for Neuroscience, 34(23), 8051-8060.

Lee, H., Samide, R., Richter, F. R., \& Kuhl, B. A. (2018). Decomposing Parietal Memory Reactivation to Predict Consequences of Remembering. Cerebral Cortex . https://doi.org/10.1093/cercor/bhy200

Maguire, E. A., Intraub, H., \& Mullally, S. L. (2016). Scenes, Spaces, and Memory Traces: What Does the Hippocampus Do? The Neuroscientist: A Review Journal Bringing Neurobiology, Neurology and Psychiatry, 22(5), 432-439.

Ngo, C. T., Michelmann, S., Olson, I. R., \& Newcombe, N. S. (2020). Pattern separation and pattern completion: Behaviorally separable processes? Memory \& Cognition. https://doi.org/10.3758/s13421-020-01072-y

Palombo, D. J., Williams, L. J., Abdi, H., \& Levine, B. (2013). The survey of autobiographical memory (SAM): a novel measure of trait mnemonics in everyday life. Cortex; a Journal 
Devoted to the Study of the Nervous System and Behavior, 49(6), 1526-1540.

Pearson, J. (2019). The human imagination: the cognitive neuroscience of visual mental imagery.

Nature Reviews. Neuroscience, 20(10), 624-634.

Pedregosa, F. (2011). Scikit-learn: Machine Learning in Python. Journal of Machine Learning

Research: JMLR, 12, 2825-2830.

Peirce, J., Gray, J. R., Simpson, S., MacAskill, M., Höchenberger, R., Sogo, H., Kastman, E., \& Lindeløv, J. K. (2019). PsychoPy2: Experiments in behavior made easy. Behavior Research Methods, 51(1), 195-203.

Richter, F. R., Cooper, R. A., Bays, P. M., \& Simons, J. S. (2016). Distinct neural mechanisms underlie the success, precision, and vividness of episodic memory. eLife, 5. https://doi.org/10.7554/eLife.18260

Robin, J. (2018). Spatial scaffold effects in event memory and imagination. Wiley Interdisciplinary Reviews. Cognitive Science, 9(4), e1462.

Robin, J., Buchsbaum, B. R., \& Moscovitch, M. (2018). The Primacy of Spatial Context in the Neural Representation of Events. The Journal of Neuroscience: The Official Journal of the Society for Neuroscience, 38(11), 2755-2765.

Robin, J., \& Moscovitch, M. (2017). Details, gist and schema: hippocampal-neocortical interactions underlying recent and remote episodic and spatial memory. Current Opinion in Behavioral Sciences, 17, 114-123.

Robin, J., Wynn, J., \& Moscovitch, M. (2016). The spatial scaffold: The effects of spatial context on memory for events. Journal of Experimental Psychology. Learning, Memory, and Cognition, 42(2), 308-315.

Schurgin, M. W., Wixted, J. T., \& Brady, T. F. (2020). Psychophysical scaling reveals a unified theory of visual memory strength. Nature Human Behaviour, 4(11), 1156-1172.

Sekeres, M. J., Winocur, G., \& Moscovitch, M. (2018). The hippocampus and related neocortical structures in memory transformation. Neuroscience Letters, 680, 39-53.

Sheldon, S., Farb, N., Palombo, D. J., \& Levine, B. (2016). Intrinsic medial temporal lobe connectivity relates to individual differences in episodic autobiographical remembering. Cortex; a Journal Devoted to the Study of the Nervous System and Behavior, 74, 206-216.

Sheldon, S., \& Levine, B. (2013). Same as it ever was: vividness modulates the similarities and differences between the neural networks that support retrieving remote and recent 
autobiographical memories. Neurolmage, 83, 880-891.

Sheldon, S., \& Levine, B. (2018). The medial temporal lobe functional connectivity patterns associated with forming different mental representations. Hippocampus, 28(4), 269-280.

Söderlund, H., Moscovitch, M., Kumar, N., Daskalakis, Z. J., Flint, A., Herrmann, N., \& Levine, B. (2014). Autobiographical episodic memory in major depressive disorder. Journal of Abnormal Psychology, 123(1), 51-60.

Sreekumar, V., Nielson, D. M., Smith, T. A., Dennis, S. J., \& Sederberg, P. B. (2018). The experience of vivid autobiographical reminiscence is supported by subjective content representations in the precuneus. Scientific Reports, 8(1), 14899.

Staresina, B. P., Cooper, E., \& Henson, R. N. (2013). Reversible information flow across the medial temporal lobe: the hippocampus links cortical modules during memory retrieval. The Journal of Neuroscience: The Official Journal of the Society for Neuroscience, 33(35), 14184-14192.

St-Laurent, M., Abdi, H., \& Buchsbaum, B. R. (2015). Distributed Patterns of Reactivation Predict Vividness of Recollection. Journal of Cognitive Neuroscience, 27(10), 2000-2018.

Thakral, P. P., Madore, K. P., \& Schacter, D. L. (2019). Content-specific phenomenological similarity between episodic memory and simulation. Memory , 27(3), 417-422.

Tibon, R., Fuhrmann, D., Levy, D. A., Simons, J. S., \& Henson, R. (2019). Multimodal integration and vividness in the angular gyrus during episodic encoding and retrieval. The Journal of Neuroscience: The Official Journal of the Society for Neuroscience. https://doi.org/10.1523/JNEUROSCI.2102-18.2018

Tulving, E. (2002). Episodic memory: from mind to brain. Annual Review of Psychology, 53, 1-25. Xie, W., \& Zhang, W. (2017). Negative emotion enhances mnemonic precision and subjective feelings of remembering in visual long-term memory. Cognition, 166, 73-83. 